\title{
artigo
}

Bernado da Silva, M.R.; Oliveira, E.P.; Tricarico, E.C.; Armada e Silva, H.C.D.; Bernado da Silva, R.B.; Santos, M.F.; Chícharo, S.C.R.;

Conhecimento sobre a sífilis e o acompanhamento de gestantes em uma clínica da familia da zona oeste do Rio de Janeiro

\section{Conhecimento sobre a sífilis e o acompanhamento de gestantes em uma clínica da família da Zona Oeste do Rio de Janeilio}

Knowledge on syphilis and follow-up of pregnant in a clinic of the family of the West Zone of Rio de Janeiro Conocimiento sobre sífilis y seguimiento de mujeres embarazadas en una clínica familiar en la zona occidental de Río de Janeiro

\begin{abstract}
RESUMO
A sífilis congênita ainda é considerada como um importante problema de saúde pública, apesar de se tratar de uma doença de fácil diagnóstico e de ser totalmente evitável. Objetivo: Identificar o conhecimento das gestantes em relação à sífilis na gestação. Metodologia: Trata-se de um estudo de campo do tipo exploratório-descritivo com abordagem qualitativa, aplicado a 15 gestantes em uma Clínica da Família na Zona Oeste do município doRio de Janeiro - RJ, Brasil. Resultados: Baixo índice de escolaridade dificultando o entendimento da doença e, na sua maioria, as gestantes fazem o tratamento, mas em contrapartida os parceiros não o realizam por diversos motivos: não são informados da doença pela companheira ou não tomam amedicação por medo. Conclusão: As gestantes afirmam ter ingressado com facilidade no pré-natal, mas ainda existem lacunas na realização do tratamento adequado e de sensibilização dos parceiros embuscar o tratamento, além de pouco conhecimento em relação à doença.
\end{abstract}

DESCRITORES: Sífilis; Conhecimento; Práticas.

\section{ABSTRACT}

Congenital syphilis is still considered an important public health problem, even though it is a disease that is easily diagnosed and totally preventable. Objective: To identify the knowledge of pregnant women in relation to syphilis during pregnancy. Methodology: This is an exploratory-descriptive field study with a qualitative approach, applied to 15 pregnant women in a Family Clinic in the west of the city of Rio de Janeiro - RJ, Brazil. Results: Low level of education making it difficult to understand the disease and, in most cases, pregnant women undergo treatment, but in contrast, partners do not perform it for several reasons: they are not informed of the disease by their partner or do not take medication for fear. Conclusion: Pregnant women claim to have entered prenatal care easily, but there are still gaps in the performance of appropriate treatment and awareness of partners to embarrass the treatment, in addition to little knowledge about the disease.

DESCRIPTORS: Syphilis; Knowledge; Understanding.

\section{RESUMEN}

La sífilis congénita todavía se considera un problema importante de salud pública, a pesar de que es una enfermedad fácil de diagnosticar y totalmente prevenible. Objetivo: identificar el conocimiento de las mujeres embarazadas en relación con la sífilis durante el embarazo. Metodología: Este es un estudio de campo descriptivo exploratorio con un enfoque cualitativo, aplicado a 15 mujeres embarazadas en una clínica familiar en el oeste de la ciudad de Río de Janeiro - RJ, Brasil. Resultados: bajo nivel de educación, lo que dificulta la comprensión de la enfermedad y, en la mayoría de los casos, las mujeres embarazadas se someten a tratamiento, pero a cambio las parejas no lo realizan por varias razones: su pareja no les informa la enfermedad o no toman medicamentos por miedo. Conclusión: Las mujeres embarazadas afirman haber ingresado fácilmente a la atención prenatal, pero todavía hay lagunas en el desempeño del tratamiento adecuado y la conciencia de las parejas para avergonzar el tratamiento, además de poco conocimiento sobre la enfermedad. DESCRIPTORES: Sífilis; Conocimiento; Prácticas.

RECEBIDO EM: 11/11/2019 APROVADO EM: 11/11/2019

\section{Maria Regina Bernardo da Silva}

Mestre em Saúde da Família e Comunidade. MBA em Gestão em Saúde. Docente da Universidade de Castelo Branco. RJ, Brasil. https://orcid.org/0000-0002-3620-3091 


\section{Eliane Pinto de Oliveira}

Enfermeira da Universidade de Castelo Branco. Pós-Graduação em Emergências e Urgências e Gestão em Saúde da Família. RJ, Brasil. https://orcid.org/00000-00016928-5655

\section{Eliane da Costa Tricarico}

Enfermeira da Universidade de Castelo Branco. Hospital Municipal Ronaldo Gazolla. RJ, Brasil. https://orcid.org/0000-00030850-8493

\section{Halene Cristina Dias Armada e Silva}

Doutoranda na UERJ. Mestre em Enfermagem. Coordenador da PAC 5.2. RJ, Brasil. https://orcid.org/0000-0003-0850-8493

\section{Raquel Bernardo da Silva}

Mestranda em Atenção Primária à Saúde na UFRJ. Preceptor da Residência de Enfermagem (PESF). RJ, Brasil. https://orcid. org/0000-0003-0850-8493

\section{Mariane Fernandes dos Santos}

Mestranda em Saúde da Família e Comunidade. Professor da Universidade de Castelo Branco. RJ, Brasil. https://orcid.org/00000003-0850-8493

\section{Sandra Conceição Ribeiro Chícharo}

Doutoranda na UFF. Enfermeira Especialista em Terapia Intensiva. Universidade Castelo Branco e Centro Universitário São José. RJ, Brasil. https://orcid.org/0000-0003-0850-8493

\section{INTRODUÇÃO}

A Organização Mundial da Saúde (OMS) fez um levantamento a respeito de sífilis e demonstra que a incidência é de cerca de um milhão de casos por ano entre as gestantes e preconiza a detecção e o tratamento oportunos destas e de seus parceiros sexuais, considerando que a infecção pode ser transmitida ao feto, com graves implicações ${ }^{(1)}$.

No Brasil e América Latina, é uma doença de notificação compulsória incluída no Sistema de Agravos de Notificação (SINAN), devendo ser investigado e notificado todo caso quer de feto vivo ou morto, filho de mãe com sífilis ${ }^{(2)}$.

Apesar de possuir o agente etiológico bem definido, formas conhecidas de transmissão e tratamentos com excelentes índices de cura, a doença é passível de eliminação, desde que a mulher e seu parceiro infectados sejam identificados e tratados antes do parto ${ }^{(1)}$.

Apesar da ampliação do diagnóstico, a maioria dos casos continua sendo detectada tardiamente. Em 2017, no Rio de janeiro, $49,5 \%$ dos casos do SINAN foram notificados de gestantes, sendo no primeiro trimestre de gestação 2557 casos, no segundo trimestre 856 , no terceiro trimestre 564 e sendo de idade gestacional ignorado, $49 \operatorname{casos}^{(3)}$.
A sífilis é uma doença transmitida pela via sexual (sífilis adquirida) e verticalmente (sífilis congênita) pela placenta da mãe para o feto. $\mathrm{O}$ contato com as lesões contagiantes (cancro duro e lesões secundárias) pelos órgãos genitais é responsável por $95 \%$ dos casos de sífilis. Outras formas de transmissão mais raras com menor interesse epidemiológico são por via indireta (objetos contaminados, tatuagem) e por transfusão sanguínea ${ }^{(2)}$.

A doença evolui de forma semelhante entre gestantes e não gestantes, podendo ser classificada conforme a forma de contágio em adquirida e congênita. A sífilis adquirida, por contágio sexual ou por hemotransfusão, pode ser: recente e tardia com mais de um ano de evolução e classificada em latente tardia e terciária. Já a sífilis congênita, adquirida por transmissão transplacentária, é classificada em recente quando diagnosticada até o segundo ano de vida e, tardia quando diagnosticada após o segundo ano devida ${ }^{(4)}$.

Os possíveis desfechos da sífilis na gestação incluem abortamento espontâneo, nascimento pré-termo e óbito perinatal em até $40 \%$ dos casos. Os nonatos sobreviventes, em mais de 50\% dos casos, apresentam-se assintomáticos, podendo, com tempo, vir a manifestar surdez, problemas visuais e até retardo mental ${ }^{(5)}$.

No Brasil, o Ministério da Saúde adotou a Estratégia Saúde da Família (ESF) como estratégia para programar ações de saúde coletiva no contexto de promoção e prevenção, juntamente com ações de cuidados, como pré-natal, para que possa fazer um diagnóstico preventivo ${ }^{(4)}$.

O pré-natal é de extrema importância para a saúde pública, e o enfermeiro tem total capacidade de conduzir as consultas. Mas, mesmo o pré-natal sendo preconizado pelo Ministério da Saúde e sendo prática dos enfermeiros nas Unidades Básicas de Saúde, foi possível verificar que ainda existem algumas dificuldades para que as gestantes cheguem ao serviço de saúde, $\mathrm{e}$ os profissionais enfermeiros, muitas vezes, não realizam a consulta de acordo com o que é estabelecido, o que pode levar a um déficit na qualidade da consulta ${ }^{(6)}$.

O pré-natal de baixo risco pode ser realizado por enfermeiro, obstetra ou não, respaldado pela Lei do Exercício Profissional da Enfermagem, Decreto n. ${ }^{\circ}$ 94.406/87. Cabe ao enfermeiro, ainda: realizar a consulta de enfermagem; realizar a prescrição de enfermagem; prescrever medicamentos, desde que estabelecido em programas de saúde pública e em rotina aprovada pela instituição de saúde; prestar 


\section{artigo}

Bernado da Silva, M.R.; Oliveira, E.P.; Tricarico, E.C.; Armada e Silva, H.C.D.; Bernado da Silva, R.B.; Santos, M.F.; Chícharo, S.C.R.;

Conhecimento sobre a sífilis e o acompanhamento de gestantes em uma clínica da familia da zona oeste do Rio de Janeiro

assistência à parturiente, puérpera e realizar educação em saúde, sendo respaldado pela Lei $.^{\circ} 7.498 / 86^{(7)}$.

O Ministério da Saúde e, conforme o Serviço de Vigilância Epidemiológica (SVE), em conformidade com a Resolução SS n. ${ }^{\circ} 41$ de 24/03/2005, preconiza que durante a assistência pré-natal toda gestante seja submetida a pelo menos dois exames de VDRL, ainda realizar novo VDRL no momento do parto para garantir ao recém-nascido a possibilidade de tratamento precoce, caso a gestante não tenha sido tratada ou tenha se reinfectado após o tratamento ${ }^{(2-7)}$.

A introdução do teste rápido no arsenal diagnóstico para melhorar a cobertura detriagem para sífilis na gravidez permite o tratamento imediato.

A Portaria do Ministério da Saúde, de 30 de dezembro de 2011, estabelece a possibilidade da utilização de teste rápido para diagnóstico de sífilis na gestante e no parceiro por profissionais capacitados nas unidades de saúde, na etapa I do diagnóstico de sífilis. O teste rápido seria utilizado quando a gestante comparece para consulta pré-natal tardiamente ou quando não há acesso ao laboratório para realização do teste do treponema pallidum ${ }^{(8)}$.

Tem-se observado falhas na assistência pré-natal, tanto na realização do teste sorológico, quanto no tratamento das gestantes e seus parceiros, indicando a perda de oportunidades importantes para a realização das ações estratégicas necessárias para o controle da sífilis ${ }^{(9)}$.

Nota-se que a captação precoce e a adesão da gestante ao pré-natal, assim como uma assistência de qualidade, dão oportunidade à essa gestante de receber informações e orientações que previnam uma gravidez não planejada e proteger-se de infecções sexualmente transmissíveis, e é neste momento que entra o acompanhamento efetuado pela equipe de enfermagem ${ }^{(10)}$.

As preocupações quanto ao cuidado das gestantes somadas aos problemas de saúde pública que a sífilis apresenta devem ser valorizados e extremamente importantes. E que a abordagem às gestantes sobre a sifilis tenha uma boa compreensão e res- ponsabilidades perante à doença, perante si e à comunidade.

A proposta de assistência integral à saúde da mulher objetiva a integralidade do atendimento, com ações educativas e clínicas, para o estabelecimento da promoção à saúde, envolvendo a enfermagem na saúde pública. Pois, cabe à enfermagem, assim como à equipe multidisciplinar, incrementar tais ações educativas, fazendo com que a gestante seja informada sobre a importância da continuidade do tratamento e do tratamento de seu parceiro para erradicação e não reincidir a doençą ${ }^{(11)}$.

Mantém-se ainda recomendaçóes para o tratamento dos parceiros, como plano de erradicação da doença. Afirma então que a transmissão sexual do Treponema pallidum ocorre somente quando as lesões da sífilis mucocutâneas estão presentes. Tais manifestações são incomuns após o primeiro ano de infecção, porém as pessoas expostas sexualmente a uma pessoa que tenha sífilis primária, latente secundária ou inicial devem ser avaliadas clinicamente e sorologicamente e tratadas ${ }^{(12)}$.

Considerando as oportunidades perdidas na prevenção e tratamento das gestantes durante a assistência pré-natal em relação à sífilis, é fundamental identificar: Qual o conhecimento das gestantes em relação a doença sífilis? Portanto, objetiva-se, com esta pesquisa, identificar o conhecimento das gestantes em relação à sífilis.

\section{METODOLOGIA}

Trata-se de um estudo de campo do tipo exploratório-descritivo com abordagem qualitativa. É descrito como método qualitativo aquele que pode ser definido e aplicado ao estudo da história, das relações, das representações, das crenças, das percepções e das opiniões, produtos das interpretações que os humanos fazem a respeito de como vivem, constroem seus artefatos e a si mesmos, sentem e pensam ${ }^{(13)}$.

O cenário de pesquisa foi uma Clínica da Família na Zona Oeste do Rio de Janeiro - RJ, e ocorreu na sala de acolhimento com as gestantes que estavam sendo acompanhadas no pré-natal. A pesquisa ocorreu de 10 a 30 de novembro de 2017, após a autorização do Comitê de Ética da SMSRJ, Parecer n. ${ }^{\circ}$ 2.127.729. Foram incluídas 15 gestantes, independentemente da idade gestacional, que após a primeira consulta, acusaram exame de VDRL positivo e que concordam em participar assinando o Termo de Consentimento Livre e Esclarecido (TCLE).

Inicialmente, algumas dificuldades aconteceram devido à localidade da clínica da família estar inserida em uma área de conflito urbano. No ano de 2018, houve 61 notificações de alerta à unidade de saúde, sendo destas, 41 alertas vermelho para fechar a unidade, dados fornecidos pelo SUBPAV do Programa Acesso Mais Seguro, por isso somente $36 \%$ das gestantes foram entrevistadas. A técnica de coleta de dados foi à entrevista semiestruturada individual ${ }^{(14)}$.

A análise de conteúdo utilizada foi a de Bardin. Para o autor, a análise de conteúdo, enquanto método, torna-se um conjunto de técnicas de análise das comunicações que utiliza procedimentos sistemáticos e objetivos de descrição do conteúdo das mensagens ${ }^{(15)}$.

A organização da análise de conteúdo, segundo Bardin, são três fases: pré-análise, exploração do material e tratamento dos resultados, inferência e interpretação. No tratamento dos resultados, ocorreu a condensação e o destaque das informações para análise, culminando nas interpretações inferenciais; com a intuição da análise reflexiva e crítica ${ }^{(15)}$.

Com a obtenção dos dados, foi efetuada uma análise do conteúdo através do levantamento das respostas obtidas pelo roteiro de perguntas e da transcrição integral das entrevistas. Os dados obtidos através das entrevistas se desdobraram em núcleos ou unidades que foram apresentados por categorias.

\section{RESULTADOS E DISCUSSÃO}

Foram entrevistadas 15 gestantes que aceitaram participar do presente estudo, às quais foi apresentado o TCLE e explicados todos os termos do mesmo e, que a partir de agora, tornam-se a amostragem 
de referência, perfazendo 36\% das gestantes portadoras de sífilis neste período.

Observa-se que $60 \%$ da incidência de sífilis está em gestantes de 20 a 25 anos, com baixa escolaridade, em sua maioria com ensino fundamental incompleto (46.7\%), sem atividade laborativa (60\%), baixa renda (60\%), possuem outros filhos (60\%), com parceiro fixo (80\%) e onde a grande maioria afirma não utilizar ou utilizar somente ocasionalmente preservativos (93,35\%).

Nesta pesquisa ficou evidente que há uma associação direta entre o desconhecimento da sífilis e seu manejo no pré-natal

devido ao baixo nível de escolaridade da gestante infectada e uma renda per capita de um a dois salários mínimos, podendo estes fatores ser marcadores de pouco acesso aos serviços de saúde, não utilização ou uso irregular de preservativo.

A distribuição dos preservativos ocorre por demanda espontânea solicitada diretamente ao responsável pela farmácia ou ao enfermeiro, mas não foi observada atividade de incentivo ao uso de preservativos para as gestantes. Este fato deixa implícito que não existe comumente a oferta $\mathrm{e}$ incentivo de utilização de preservativos à gestante,

Tabela 1. Perfil socioeconômico e convivência marital das gestantes em uma Clínica da Familia na Zona Oeste. Rio de Janeiro, RJ, Brasil, 2017.

IDADE

Idade

20 a 25

26 a 35

36 a 40

NIIVEL DE ESCOLARIDADE

Ensino fundamental incompleto

Ensino fundamental completo

Ensino médio incompleto

Ensino médio completo

\section{ATIVIDADE LABORATIVA}

Sim

Não

RENDA FAMILIAR

Sem renda

Menos de um salário mínimo

Um a dois salários mínimos

Três ou mais salários mínimos

PARIDADE

Nenhum

Sim

PARCEIRO FIXO

Sim

Não

USA PRESERVATIVO NAS RELAÇÕES

Sim

Não

Às vezes

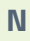

N

09

04

02 como se por estar gestante não houvesse a prática sexual. Entretanto, como demonstra nesta pesquisa, a reincidência ocorre e a gestante não entende como ou porquê de ter que refazer seu tratamento ${ }^{(16)}$.

Alguns autores afirmam que a não utilização do preservativo está associada aos significados atribuídos aos atos sexuais, ao próprio preservativo e ao processo de negociação do seu uso, inseridos num conjunto de códigos, normas e expectativas coletivas. Entretanto, o perigo resulta do fato das relações sexuais envolverem parceiros pouco conhecidos e serem baseadas numa confiança frágil, ou seja, a mulher "confia” em seu parceiro e a não utilização do preservativo passaria a ele essa confiança. Se o parceiro não tem relação sexual com mais ninguém, por que ela deveria utilizá-lo? Assim, considera-se dispensável o seu uso quando a prática sexual envolve parceiros (as) habituais, em quem se deposite confiança, sejam eles solteiros ou casados, no contexto de relações formalmente monogâmicas ou não ${ }^{(17)}$.

Outros argumentos apresentados para explicar o não uso do preservativo passam pelo receio de abordar este assunto, sobretudo no caso das relações em que ele nunca fora usado, pela ideia de que os preservativos provocam alergias, que se rompe com facilidade e incomodam, pela falta de vontade de usá-los, pelo fato de atrapalharem pretensões relativas à procriação ou ainda de minimizarem o usufruto de dinheiro ou bens investidos em troca da prática sexual ${ }^{(18)}$.

Em relação ao conhecimento da doença sífilis das 15 gestantes, 93,35\% ${ }^{(14)}$ afirma ter iniciado o pré-natal no primeiro trimestre da gravidez, e nenhuma delas encontrou dificuldade para a marcação da primeira consulta. Em sua totalidade, afirmam que foram informadas sobre a necessidade do exame de sífilis na primeira consulta do pré-natal. Sobre o conhecimento da doença sífilis, $80 \%$ afirma saber ou conhecer superficialmente a doença, sendo a maior fonte de conhecimento a leitura $(39,5 \%)$, seguida por informações advindas de conhecidos e dos profissionais da área de saúde, ambos com 26,7\%.

$A$ atenção pré-natal tem entre seus pro- 


\section{artigo}

Bernado da Silva, M.R.; Oliveira, E.P.; Tricarico, E.C.; Armada e Silva, H.C.D.; Bernado da Silva, R.B.; Santos, M.F.; Chícharo, S.C.R.;

Conhecimento sobre a sífilis e o acompanhamento de gestantes em uma clínica da familia da zona oeste do Rio de Janeiro

tocolos a triagem da sífilis na gestante e o consequente tratamento da mulher quando apresentam testes positivos. Assim, a Clínica da Família torna-se um espaço ideal para o controle da sífilis congênita, principalmente no que se refere ao diagnóstico precoce e tratamento adequado dos casos com VDRL positivo, bem como de seus parceiros sexuais, que devem receber cuidados concomitantes.

Entre as entrevistadas, 53,7\% informou ter conhecimento da transmissão da sífilis, mas poucas utilizam preservativo, somente uma disse utilizar no seu dia a dia. Esse fato contribui para disseminação da doença.

Portanto, as consultas não devem ser somente compostas por anamnese e solicitação de exames, mas uma oportunidade para a gestante retirar dúvidas e do profissional responsável transmitir informações relevantes à saúde da mulher e do seu bebêt $\hat{e}^{(19)}$. As gestantes, na sua maioria $(66,7 \%)$, informaram ter feito três doses de Benzetacil, seguidas por $33,3 \%$ de duas doses. Quanto à informação aos parceiros, $80 \%$ afirmou que avisou que era portadora da doença. Destes homens informados, $40 \%$ não buscou tratamento. Os que foram informados e não fizeram o tratamento, o motivo alegado foi de não querer ir à Clínica da Família tomar a medicação e medo.

A ausência dos homens nas Clínicas da Família demonstra o quanto a sociedade ainda é machista e preconceituosa, tendo ainda a concepção de que a gravidez e a criação dos filhos são de inteira responsabilidade da mulher. O número de casos de parceiros não tratados é superior ao número de parceiros tratados, o que mostra a dificuldade na queda de cadeia de transmissão e indica o acompanhamento inadequado das gestantes ${ }^{(20)}$.

Observa-se que das 15 gestantes entrevistadas, seis parceiros não foram tratados, ficando bem aquém do esperado pelo Ministério da Saúde.Em relação ao tratamento, a terapia medicamentosa intramuscular contribui para a resistência pela dor na administração e a quantidade de doses necessárias para a conclusão do tratamento ${ }^{(21)}$.

É importante para evitar a reinfecção durante a gravidez, principalmente em locais de alta prevalência da sífilis, que os parceiros sejam comunicados, localizados e tratados, pois, além de ocasionar a reincidência na parceira, permanecem com reservatórios de IST na população geral.

Durante o acompanhamento das gestantes, o Ministério da Saúde propõe assistência suplementar a homens e mulheres para receberem orientaçôes sobre a forma de prevenção da transmissão vertical, tendo como principal forma de prevenção a identificação e tratamento das gestantes portadoras de sífilis ${ }^{(22)}$.

As questões abertas foram caracterizadas por três categorias diversas, como demonstradas a seguir.

\section{0 conhecimento e entendimento das gestantes sobre a doença sífilis e seu tratamento}

Observou-se que $60 \%$ das entrevistadas relata desconhecer sobre a doença sífilis e quanto ao tratamento. Apesar de responderem anteriormente nas questões fechadas que conheciam a doença, seus relatos foram bem frágeis, demonstrando pouco conhecimento a seu respeito dadoença.

$H, M$ "Não entendo bem. Sei que é uma DST transmissivel e quanto ao tratamento logo que descobri, iniciei o tratamento com Benzetacil."

$J$, L "Não sei direito, masé uma doença grave para o bebê, tomei injeção."

$A, B, C$, "Não consegue entender a doença."

Estudo similar feito em Sobral, no Ceará, afirma que nas falas das participantes fica demonstrado o desconhecimento sobre a doença, as formas de prevenção e tratamento, sendo um dos fatores que dificultam a adesão do casal ${ }^{(23)}$.

Outra questão é que o pouco conhecimento das mulheres quanto à sífilis pode ter sido adquirido somente após a confirmação da doença. Isso leva a acreditar que antes do diagnóstico as mulheres não possuíam conhecimento algum acerca da sífilis e passam a se interessar pela temática após o diagnóstico ${ }^{(24)}$.

Das entrevistadas, $40 \%$ relatou conhecimento sobre a doença e tratamento, mas apesar do conhecimento, tiveram a doença durante a gravidez.

I, $K$. "Ah, é uma doença transmissivel pelo sexo, sangue e o tratamento que fiz é com Benzetacil."

EF. "Eu entendo. Só não sei porquêprecisei repetir o tratamento feito antes." $\mathrm{Ne}$ O."É uma doença transmissivel pelo sangue, sexo e placenta e tomei 3 doses de Benzetacil durante três semanas."

Quanto à transmissão da doença, pôde-se notar existente confusão entre a sífilis adquirida e a sífilis congênita, não sendo observada uma distinção específica pela maioria das gestantes.

Em outro estudo, também realizado no Ceará na cidade de Crato, as enfermeiras colocaram em destaque alguns pontos frágeis quanto a não adesão ao tratamento, que estiveram relacionados aos parceiros de gestantes, como: nível de escolaridade, desconhecimento da doença, atividades laborais, nível de relacionamento com a gestante e ausência no pré-natal ${ }^{(25)}$.

Este desconhecimento da doença é atribuído, ainda, à baixa qualidade do pré-natal devido a falhas no serviço de saúde e de políticas de avaliação da qualidade da assistência prestada, notada com a não realização do rastreamento das gestantes, ausência do retorno das gestantes à unidade, não adesão e tratamento dos parceiros, não realização do protocolo de sete consultas durante a gestaçãa ${ }^{(26)}$.

Entretanto, observa-se que muitas mulheres acreditam que apenas uma relação desprotegida não é suficiente para adquirir alguma IST. Acredita-se também que a promiscuidade seja a provável contaminação da mulher pelo seu parceiro ${ }^{(25)}$.

O conhecimento das gestantes a respeito da sífilis não foi entendido adequadamente durante o pré-natal, uma vez que as consultas são o espaço que as gestantes têm para sanar as dúvidas e os profissionais para praticar a educação em saúde.

\section{Conhecimento sobre como evitar a doença Sífilis}

Nesta categoria, analisa-se o conhecimento sobre como evitar a doença sífilis 
nas falas das entrevistadas e obteve-se as seguintes respostas:

$A, B$, e L"Sei que precisamos usar camisinha nas relaçôes e não ter muitos parceiros sexuais, né?"

E, I e N" Usando camisinha nas relações sexuais evita a sifilis e o tratamento é com injeção de Benzetacil." C, Je M "Não conheço essa doença, mas sei que toma injeção no tratamento."

Sabe-se que a gestante positiva e seu parceiro devem ser tratados e acompanhados pelo serviço de saúde, e recomenda-se o uso de camisinha mesmo após o tratamento. Entretanto, as mulheres necessitam receber informações durante a assistência pré-natal, tornando-as detentoras dos meios que levem a prevenção contra a reincidência da doença.

Como observado neste estudo, o parceiro ocasionalmente não é informado, fazendo com que a utilização do preservativo e o tratamento do parceiro sejam fatores determinantes para a cura eficaz da gestante. Se considera inaceitável a ocorrência da sífilis congênita nos dias de hoje, onde o rastreamento sorológico é obrigatório no acompanhamento pré-natal e o tratamento e a prevenção adequados são perfeitamente capazes de evitar a infecção do concepto e a reinfecção materna. Estas medidas são simples, amplamente disponíveis, de baixo custo e grande impacto no controle da doençą ${ }^{(27)}$.

Entretanto, observa-se pelas falas das entrevistadas que, apesar de suas afirmações em saber como evitar a doença, constatou-se reincidência de sífilis, as mesmas encontram-se em tratamento, pois estão portadoras da doença. Neste sentido, chama-se a atenção para a importância de métodos contraceptivos, como preservativo feminino, que empoderam a mulher facilitando sua autonomia, principalmente, no que se refere à escolha pelo sexo seguro e dupla proteção.

\section{As consequências do tratamento inadequado}

$A, F, e$ L " $A$ enfermeira disse que não fazendo o tratamento o bebê nasce doente com sifilis."

\section{$B, F, I$ e $O$ "O bebê nasce com a do- ença e pode morrer." \\ C e D "Não sei o que pode ocorrer."}

Das entrevistadas, 63\% (09) afirmou saber que seus filhos poderiam desenvolver sequelas ou até mesmo morrer, caso nascessem com o diagnóstico da doença, mas não sabiam ao certo relatar quais seriam essas complicações, preocupa-se assim acerca das formas como são repassadas estas informações para elas.

A falta de tratamento adequado da gestante portadora de sífilis poderá desenvolver consequências no neonato, como: baixo peso, prematuridade, óbito fetal, complicações agudas, neurológicas, deformidades, alterações dentária e óssea ${ }^{(28)}$.

O Ministério da Saúde acrescenta que dentre os principais sintomas estão: lesões cutâneo-mucosas (exantema maculoso), principalmente na face e extremidades; lesões bolhosas; fissuras periorais e anais. $\mathrm{Na}$ sífilis congênita tardia, as lesões são irreversíveis caracterizadas por: fronte olímpica, palato em ogiva, tíbia em sabre, dentes de Hutchinson e molares em amora ${ }^{(3)}$.

De acordo com OMS, os usuários dos serviços de saúde devem receber informação sobre a sífilis e serem convencidos de que a prevenção e o tratamento podem resultar em benefícios importantes para a saúde materna e da criança que nascerá. Destacando ainda que abordagens comunitárias podem ser importantes para informar o público vulnerável e estimular a procura de meios para a detecção da sífilis ${ }^{(11)}$.

No entanto, não se pode deixar de levar em consideração a baixa escolaridade das mães, o que nos leva a considerar que elas até podem ter recebido as informações referentes à doença, porém não conseguem assimilar ou entender este aprendizado.

Igualmente é importante ressaltar que as instituições de saúde pública geralmente têm dificuldade em produzir programas educativos voltados para a população que não têm educação formal, o que contribui para a não concretização do aprendizado de informações indispensáveis à prevenção, tornando-as mais vulneráveis a contrair muitas doenças ${ }^{(29)}$.

\section{CONCLUSÃO}

A sífilis é uma das infecções sexualmente transmissíveis que causam maiores danos às gestantes e filhos. Apesar de se conhecer o agente etiológico, seu modo de transmissão, ter-se o tratamento eficaz e de baixo custo, com excelentes possibilidades de cura, ainda persiste como um grave problema de saúde pública.

Através deste estudo foi possível concluir que as gestantes entrevistadas não possuíam conhecimento suficiente para suprir suas necessidades quanto à doença. Apesar de saberem, ou afirmarem ter recebido informações relacionadas à doença, demonstraram dificuldade de se expressar. Este fato é decorrente de dificuldades associadas ao contexto social no qual estão inseridas, como: baixa condição socioeconômica, baixo nível de escolaridade, pouca adesão a parceiros fixos e desconhecimento prévio acerca da doença.

Por outro lado, pode-se afirmar que, apesar da cobertura dos programas da rede de saúde quanto ao pré-natal, a totalidade de gestantes afirma ter ingressado com facilidade na unidade básica, mas ainda existem lacunas na realização do tratamento adequado e de sensibilização dos parceiros em buscar o tratamento.

Nota-se a necessidade de uma maior atenção à gestante durante o pré-natal, ações educativas à comunidade em que estão inseridas para poder auxiliar nesse entendimento e minimizar sua reincidência. E não podemos também deixar passar em branco a violência local que pode também dificultar o retorno dessas mulheres à unidade de saúde.

Por fim, pode-se afirmar que existe uma lacuna entre a intenção e o gesto, principalmente no que se refere à transmissão de conhecimento por parte dos profissionais. Fica a sugestão para se aproveitar todas as oportunidades de comparecimento dessa gestante e que sejam desenvolvidas ações educativas voltadas para a ampliação do conhecimento e sensibilização da gestante a respeito de sífilis e a sensibilização da presença do companheiro nas consultas de pré-natal, sempre que possível. - 


\section{artigo}

Bernado da Silva, M.R.; Oliveira, E.P.; Tricarico, E.C.; Armada e Silva, H.C.D.; Bernado da Silva, R.B.; Santos, M.F.; Chícharo, S.C.R.i Conhecimento sobre a sífilis e o acompanhamento de gestantes em uma clínica da familia da zona oeste do Rio de Janeiro

\section{REFERÊNCIAS}

1. Ministério da Saúde (BR). Boletim epidemiológico [Internet]. 2015 [acesso em 02 set 2016]. Disponivel em: http://www.aids. gov.br/sites/default/files/anexos/publicacao/2015/57978/_P_ boletim_sifilis_2015_fechado_pdf_p18327.pdf.

2. Organização Pan-Americana da Saúde. Dados sobre Sífilis [Internet]. 2014 [acesso em 10 dez 2016]. Disponivel em:

http://www.paho.org/bra/index.php?option=com_content\&view=article\&id=4955:dados-17- paises-territorios-americas-apontam-eliminacao-transmissao-mae-filho-hiv- sifilis\&ltemid=816.

3. Ministério da Saúde, Secretaria de Vigilância em Saúde, Coordenação-Geral de Desenvolvimento da Epidemiologia em Serviços (BR). Guia de Vigilância em Saúde : volume 2/ Ministério da Saúde, Secretaria de Vigilância em Saúde [Internet]. 1. ed. atual. - Brasília : Ministério da Saúde, 2017 [acesso em 10 dez 2019]. Disponivel em: http://bvsms.saude.gov.br/bvs/publicacoes/guia vigilancia_saude_volume_2.pdf.

4. Signorini DJHP, Monteiro MCM, Sá CAM, Sion FS, Leitão-Neto HG, Lima DP. Prevalência da co-infecção HIV - sífilis em um hospital universitário da cidade do Rio de Janeiro no ano de 2005. Rev Soc Bras Med Trop. 2007;40(3):282-5.

5. Araújo CL, Shimizu HE, Souza AIA, Hamman EM. Incidência da sífilis congênita no Brasil e sua relação com a Estratégia Saúde da Família. Rev. Saúde Pública. 2012; 46(3): 479-86.

6. Brasil, Ministério da Saúde, Assistência pré-natal. $3^{a}$ ed. Brasília: (DF); 2000, disponivel em <http://bvsms.saude.gov.br/bvs/ publicacoes/cd04_11.pdf>, acesso em dezembro de2016.

7. Ministério da Saúde, Departamento de Ações Programáticas Estratégicas, ÁreaTécnica de Saúde da Mulher (BR). Diretrizes gerais e operacionais da Rede Cegonha. Brasília: Ministério da Saúde, 2011

9. Saraceni V, Leal MC, Hartz ZMA. Avaliação de campanhas de saúde com ênfase na sífilis congênita: uma revisão sistemática. Rev. Bras. Saúde Mater. Infant. 2005; 5(3):263-73.

10. Domingues RMS, Hartz ZMA, Leal MC. Avaliação das ações de controle da sífilis e do HIV na assistência pré-natal da rede pública do município do Rio de Janeiro, Brasil, Rev. Bras. Saúde Matern. Infant. 2012 jul./set.; 12(3):269-280.

11. Vinha V, Almeida MC, Volpi CL. Sífilis, orientação e assistência de enfermagem em saúde pública em u m hospital escola. Rev bras enferm. 70-80.

12. Center for Deasease Control and Prevent. Sexually Transmitted Diseases Treatment Guidelines [Internet], 2015 [acesso em 03 set 2016]. Disponível em: http://www.cdc.gov/mmwr/pdf/rr/ rr6403.pdf.

13. Minayo MCS. O desafio do conhecimento: Pesquisa Qualitativa em Saúde. 12. ed. São Paulo: Hucitec-Abrasco; 2010.

14. Comitê Internacional da Cruz Vermelha. Programa Acesso Mais Seguro [Internet]. 2016 [acesso em 02 fev 2018]. Disponivel em: https://www.icrc.org/pt/document/o-programa-acesso-mais-seguro.
15. Bardin L. Análise de Conteúdo. Lisboa: Edições 70; 2009.

16. Lopes MH. Avaliação da implementação das ações de Prevenção da Transmissão Vertical de Sífilis no Pré-Natal em Unidades de Saúde da Família de Cuiabá. Dissertação apresentada com vistas à obtenção do título de Mestre Modalidade Profissional em Saúde Pública. 2010

17. Gune E. Momentos liminares: dinâmica e significados no uso do preservativo. Anál. Social. 2008 abr; 187.

18. Organização Pan-Americana da Saúde. Dados sobre Sífilis [Internet]. 2014 [acesso em 02 dez 2016]. Disponivel em: http:// www.paho.org/bra/index.php?option=com_content\&view=article\&id=4955:dados-17- paises-territorios-americas-apontam-eliminacao-transmissao-mae-filho-hiv- sifilis\&ltemid=816.

19. Ministério da Saúde, Secretaria da Vigilância em Saúde (BR). Programa Nacional de DST e Aids. Protocolo para a prevenção de transmissão vertical de HIV e sífilis. Brasília: Ministério da Saúde, 2006.

20. Signorini DJHP, Monteiro MCM, Sá CAM, Sion FS, Leitão Neto HG, Lima DP. Prevalência da co-infecção HIV - sífilis em um hospital universitário da cidade do Rio de Janeiro no ano de 2005. Rev Soc Bras Med Trop. 2007;40(3):282-5.

21. Araújo CL, Shimizu HE, Souza AIA, Hamman EM. Incidência da sífilis congênita no Brasil e sua relação com a Estratégia Saúde da Família, Rev. Saúde Pública, 2012; 46(3): 479-86.

22. Ministério da Saúde (BR). Assistência pré-natal. 3. ed. Brasília: Ministério da Saúde, 2000.

23. Secretaria de Estado de Saúde do Rio de Janeiro (BR). Informe Epidemiológico [Internet]. 2016 [acesso em 03 set 2017]. Disponivel em: https:// www.informacaoemsaude.rj.gov.br\%2Fdocman\%2Fdst-aids\%2F12975-informe-epidemiologico-sifilis-materna-e-congenita-n-1-2016.html\&usg=AOvVaw30Bkr1qDbUHLArJj7lemAT.

24. Serviço de Vigilância Epidemiológica (BR). Sífilis Congênita e Sífilis na Gestação. Rev Saúde Pública. 2008;42(4):768-72

25. Ministério da Saúde, Departamento de Ações Programáticas Estratégicas, ÁreaTécnica de Saúde da Mulher (BR). Diretrizes gerais e operacionais da Rede Cegonha. 2011.

26. Ministério da Saúde (BR). Portaria n. ${ }^{\circ}$ 3.242, de 30 de dezembro de 2011. Dispõe sobre o Fluxograma Laboratorial da Sífilis e a utilização de testes rápidos para triagem da sífilis em situações especiais e apresenta outras recomendações. 2011.

27. Saraceni V, Leal MC, Hartz ZMA. Avaliação de campanhas de saúde com ênfase na sífilis congênita: uma revisão sistemática. Rev. Bras. Saúde Mater. Infant. 2005; 5(3):263-73.

28. Domingues RMS, Hartz ZMA, Leal MC. Avaliação das ações de controle da sífilis e do HIV na assistência pré-natal da rede pública do município do Rio de Janeiro, Brasil. Rev. Bras. Saúde Matern. Infant. 2012 jul./set.; 12(3):269-280.

29. Organização Mundial de Saúde. Eliminação mundial da sífilis congênita: fundamento lógico e estratégia para ação. Washington, 2008. 